\title{
Tarsal Tunnel Syndrome (TTS) in Fibromyalgia patients
}

Mohamed Ahmed Hefny', Shaimaa mohamed refaat okasha ${ }^{2}$, Mai Abdulrahim Abdullatif ${ }^{2}$, Marwa orabi ${ }^{4}$, Asser Sallam ${ }^{5}$, Nermeen A. Moneim $^{2}$ and Mai Mohamed Abdelnaby ${ }^{2}$

${ }^{1}$ Professor of Physical Medicine, Rheumatology and Rehabilitation. Faculty of Medicine - Suez Canal University, Ismailia, Egypt ${ }^{2}$ Lecturer of Physical Medicine, Rheumatology and Rehabilitation. Faculty of Medicine - Suez Canal University, Ismailia, Egypt

${ }^{3}$ Lecturer of Neurology. Lecturer of Neurology, Department of Neuropsychiatry. Faculty of Medicine - Suez Canal University Hospital, Ismailia, Egypt

${ }^{4}$ Lecturer of Lecturer of Orthopedic Surgery. Department of Orthopedic Surgery. Faculty of Medicine - Suez Canal University, Ismailia, Egypt

\section{Introduction}

Fibromyalgia (FM) is a syndrome of unknown etiology, characterized by chronic widespread pain, and increased tenderness on palpation. Some other disorders like irritable bowel syndrome, disrupted sleep, stiffness, fatigue, psychological disorders and cold intolerance, migraine and premenstrual syndrome are also related to this specific syndrome [1]. Fibromyalgia patients are typically affected with substantial disability and emotional distress [2].

Fibromyalgia has an estimated prevalence of $2 \%$ of the United States adult general population and its prevalence increases in women, peaking between ages 54 and 64 years and then decreasing. The prevalence in men is fairly constant across ages [3]. Paresthesia and sensory alterations are problematic complaints seen in $>80 \%$ of $\mathrm{FM}$ patients. They may be confused with entrapment neuropathies and radiculopathies. The mechanism of paresthesia in FM is not exactly known. However, paresthesia is considered to be a result of an abnormal sensory perception occurring due to central sensitization [3]. However, peripheral mechanisms in muscles and nerves have not been excluded as possible sources of pain and paresthesia in fibromyalgia [4].

Posterior tarsal tunnel syndrome (TTS) is characterized by symptoms over the plantar aspect of the foot, which exacerbate either on standing, prolonged walking, or wearing tight footwear. The symptoms of TTS include pain, numbness, tightness, or burning sensations over the sole and tend to vary in severity and distribution between the patients to such an extent that it is recommended to consider the possibility of TTS in all those who present with sensory symptoms in the sole [5].

Paresthesia of the foot and local tenderness at the tarsal tunnel was significantly more prevalent in FM patients than in healthy control subjects [4].

It seems that patients with fibromyalgia experience a lower quality of life as compared to the general population. Many patients provided a broad range of areas on which FM had a significant and negative impact on their lives including mood, family, social relationships, and work efficiency. Some of them stated that their disease affects every aspect of their life. Patients also discussed the need to push themselves to do activities and indicated that the fatigue they experience makes even simple tasks, such as walking or standing, difficult [6].

The purpose of this study was to estimate the frequency of tarsal tunnel syndrome (TTS) in fibromyalgia (FM) patients and study its impact on their quality of life.

\section{Subjects and Methods}

The study included 45 subjects diagnosed with Fibromyalgia syndrome according to the American College of Rheumatology (ACR) criteria [7], attending the Rheumatology \& Rehabilitation outpatient clinics at Suez Canal University Hospitals. All the patients were females, their ages ranged from 25 to 46 years.

Thirty apparently healthy females served as a control group for the nerve conduction study normative data.

Neurological, vascular, endocrinal or metabolic disorders were excluded from the study. Patients with chronic ankle injuries were also excluded. To minimize the risk factor of occupational impact, housewives were included in the study, of moderate socio-economic standard. None of the patients or controls performed sustained prolonged long standing activities.

\section{History \& clinical examination}

The history explored the foot pain characteristics, distribution of the pain (lateral sole, medial sole, heel, or more than one location), aggravating, and relieving factors of the pain. Medical history of diabetes, arthritis, and claudication was obtained. Physical examination included the recording of the weight, height, palpation of distal pedal pulses, elicitation of Tinel's sign over the TT [8]. The body mass index (BMI) was calculated [9]. The examination also included the elicitation of deep tendon reflexes and sensory evaluation of the soles at four sites (heel, the base of the toes, and the plantar aspect of great toe) [10]. The distribution of sensory symptoms and signs were mapped on a template and grouped as per the location into the medial sole, lateral sole, only heel and more than one site.

\section{Assessment of functional status of FM patients}

The Arabic version of Fibromyalgia impact questionnaire (FIQ-A) [11] was completed by every patient.

The FIQ-A is an assessment and evaluation instrument developed to measure FM patient status, progress and outcomes. It has been designed to measure the components of health status that are believed to be most affected by FM [11].

${ }^{\star}$ Correspondence to: Shaimaa mohamed refaat okasha lecturer of physical medicine, Rheumatology and Rehabilitation. Faculty of medicine Suez canal university hospital, Ismaillia, Egypt. killo 4.5 round road Suez canal university hospital, Department of physical medcine, Rheumatology and Rehabilitation. ismaillia, Egypt, Tel: 00201151338558; E-mail: shaymaa_ramdan@med.suez.edu.eg

Received: July 01, 2018; Accepted: July 10, 2018; Published: July 12, 2018 
The FIQ is composed of 10 items. The first item contains 11 questions ("a" through "k") related to physical functioning - each question is rated on a 4 point Likert type scale. Items 2 and 3 ask the patient to mark the number of days they felt well and the number of days they were unable to work (including housework) because of fibromyalgia symptoms. Items 4 through 10 are horizontal lines, $10 \mathrm{~cm}$ in length, in which the patient rates work difficulty, pain, fatigue, morning tiredness, stiffness, anxiety and depression.

\section{Electrodiagnosis}

Motor nerve conduction study was performed by one of the investigator (MD) on Neurosoft, Neuro-MEP-Micro NCS \& EMG machine. Surface disk electrodes were used for recording the response. The MPN [12], LPN [13], ICN [14] tibial and sural [15] nerves were evaluated on both the sides. The sural nerve was stimulated on the calf slightly lateral to the midline in the lower third of the leg behind $140 \mathrm{~mm}$ proximal to the lateral malleolus and recorded by placing the recording electrode behind the lateral malleolus. The tibial nerve was stimulated behind medial malleolus $80 \mathrm{~mm}$ proximal to the active electrode, placed over the most prominent part of the belly of abductor hallucis muscle and in the popliteal fossa.

The PTN was stimulated at the ankle (proximal to the flexor retinaculum, $1 \mathrm{~cm}$ posterior to the medial malleolus) and the response was recorded $80 \mathrm{~mm}$ distal to the stimulation point over the abductor hallucis muscle (MPN) [12]. The PTN stimulated sequentially, at the site as mentioned above and responses were recorded over abductor digiti quinti (ICN) [14] halfway between the tip of the lateral malleolus and sole and stimulated behind medial malleolus $80 \mathrm{~mm}$ proximal to the active electrode, placed as while testing abductor hallucis muscle, known as Fu's method and over flexor digiti minimi brevis musclealong the midpoint of the inferolateral edge of the $5^{\text {th }}$ metatarsal (LPN), the stimulation point being the same as was for the recording from Abductor Hallucis muscle [12]. During the electrodiagnostic study, the skin temperature of the limb was maintained between $29^{\circ} \mathrm{C}$ and $31^{\circ} \mathrm{C}$. In the case of no response or a low CMAP amplitude, the procedure was repeated after altering the electrodeposition and or the interelectrode distance; best response was included in the analysis.

\section{The following electrodiagnostic parameters were noted as follows}

Terminal latency or distal latency $[1,15]$ stimulus artifact to the take-off of the negative peak of the motor response, in milliseconds (ms). For measuring the latency of ICN CMAP, the initial negative peak, if present, was ignored

CMAP amplitude (a): [15] measured from the baseline to negative peak, millivolts $(\mathrm{mV})$

Duration of the negative peak of CMAP (d): [15] from the onset to the first base line, cross of negative peak

Sural nerve sensory action potential latency

SNAP amplitude

Tibial nerve motor conduction velocity between the knee and the ankle.

Based on the above observations, following parameters were further calculated:

1. Medial plantar latency (MPl)

2. Lateral plantar nerve latency $(\mathrm{LPl})$

3. Amplitude of the medial plantar CMAP (MPa)

\section{Amplitude of the LPN CMAP (LPa)}

\section{ICN latency (ICNl)}

\section{Amplitude of the ICN CMAP (ICNa)}

\section{Duration of the ICN CMAP (ICNd).}

Values of terminal latency, duration, and amplitude of the CMAP were used for identifying the demyelination or axonopathy in peripheral nerves. The former reflects in MNCS as delayed distal latency in prolonged duration of the negative peak of CMAP; the later reflects a reduction in the amplitude of negative peak [16,17]. Accordingly, nerves with delayed DL, ( $>2$ standard deviation [SD] of the upper limit of normal[ULN]) with or without prolongation of the duration of the CMAP, were considered to have "probable demyelination" while nerves showing low CMAP amplitude with normal DL were considered to be having "probable axonopathy."

\section{All the following criteria were included in the study:}

1. The presence of sensory symptoms (pain, tingling, burning, tightness, and jabs) restricted to the plantar aspect of the foot. The symptoms, not relieved by analgesics, should have persisted for more than 4 weeks, preceding the day of inclusion.

2. Symptoms should be persisting most of the day, with aggravation on standing, walking, prolonged standing or wearing footwear

3. Absence of tenderness at the medial calcaneal tuberosity and middle portion of plantar fascia, low back pain, pain in legs, diabetes, rheumatoid arthritis, history of surgery in the symptomatic limb or spinal surgery, foot deformity, gout, family history of burning feet, signs of distal symmetric neuropathy (graded distal sensory loss, and absent ankle jerks), and abnormal dorsalis pedis pulsations

4. Should not have received local steroids or analgesic injections.

Those who had abnormal knee and ankle tibial motor conductions (slow conduction velocity, conduction block, or temporal dispersion (16)) and sural SNAPs (low amplitude) were excluded.

\section{Results}

This study was carried out on 75 housewives: 45 female subjects suffering from fibromyalgia and 30 healthy female volunteers as control group. There were non-significant differences between both groups as regards their age and BMI (Table 1).

Regarding Prevalence of TTS in FM patients (Table 2) in our study, $17(37.8 \%)$ of FM patients and $3(10 \%)$ of control subjects were suffering from foot paresthesia. 15 (33\%) of FM patients and 5 (16\%) of control subjects were suffering from foot burning sensation By clinical examination, only 11(24\%) FM patients and 4(13\%) of control subjects had positive signs for Tinel sign.

Electrophysiologically TTS was found in 8 (17.7\%) of FM subjects and in $4(13.3 \%)$ in control subjects (Table 3$)$. The frequency of TTS among FM and control subjects was evaluated. FM patients had a significantly higher frequency of TTS as compared to the control group $(p<0.001)$. In our study, there was a highly statistically significant correlation between the presence of TTS and FIQ score of FM patients (Table 4). Patients with TTS had their FIQ scores ranging from 30 to 79 (59.41 \pm 14.4$)$. While patients without TTS had their FIQ scores ranging from 24 to $51(37.5 \pm 7.4)$. We found the difference highly statistically significant as regards the FIQ score between the two subgroups $(p<0.001)$. 
Table 1. Demographic data in both groups

\begin{tabular}{|c|c|c|c|}
\hline & Number & BMI (mean \pm SD) & $32 \pm 1.5$ \\
\hline Study group & $45(60 \%)$ & $31 \pm 1.3$ & female \\
\hline Control group & $30(30 \%)$ & female \\
\hline Total & 75 & p-value $=1.1$ \\
\hline
\end{tabular}

Table 2. Tarsal tunnel symptoms in both groups

\begin{tabular}{|c|c|c|c|c|c|}
\hline Tarsal tunnel symptoms & \multicolumn{3}{|c|}{ Control group } \\
\hline Pain & 30 & $(66.6 \%)$ & 4 & $(13 \%)$ \\
\hline Numbness and paresthesia & 17 & $(37.8 \%)$ & 3 & $(10 \%)$ \\
\hline Burning sensation & 15 & $(33 \%)$ & 5 & $(16 \%)$ \\
\hline Tinel sign & 11 & $(24 \%)$ & 4 & $0.000 *$ \\
\hline
\end{tabular}

Table 3. electrodiagnosis of Tarsal tunnel syndromes in both groups

\begin{tabular}{|c|c|c|}
\hline & Study group & Control group \\
\hline With TTS & $8(17.7 \%)$ & $4(13.3 \%)$ \\
\hline Without TTS & $37(82.3 \%)$ & $26(86.7 \%)$ \\
\hline Total & 45 & 30 \\
\hline
\end{tabular}

Table 4. Correlation between positive tarsal tunnel diagnosis and Fibromyalgia impact questionnaire in study group

\begin{tabular}{|c|c|c|}
\hline & & FIQ scores (mean \pm SD) \\
\hline With TTS & $8(17.7 \%)$ & ranging from $30-79(59.41 \pm 14.4)$ \\
\hline Without TTS & $37(82.3 \%)$ & ranging from $24-51(37.5 \pm 7.4)$. \\
\hline Total & 45 & \\
\hline
\end{tabular}

\section{Discussion}

Fibromyalgia can have a substantial impact on patients' lives. A survey of women utilizing the National Fibromyalgia Association Web site reported substantial disability with fibromyalgia (18). A high prevalence of undetected TTS in patients with FM has been reported [4].

The purpose of this study was to estimate the frequency of tarsal tunnel syndrome (TTS) in fibromyalgia (FM) patients. This study may contribute to shed some light on the pathogenesis of pain and paresthesia among TTS patients.

In our study, the prevalence of TTS in FM patients was $17.7 \%$, while it was $13.3 \%$ in the control subjects. This difference was highly statistically significant $(p<0.001)$. This is much higher than the prevalence reported by Yo and $\mathrm{Na}$, in which was $18.4 \%$ in $\mathrm{FM}$ patients and $3.3 \%$ in control subjects, and the difference did not reach statistically significant levels [4].

Also, Shookster and colleagues found the prevalence of TTS was higher than control subjects, although it did not reach statistically significant levels $[18,19]$.

Patient's job could be a causative agent for the occurrence of TTS. To avoid the effect of occupation, all subjects involved in this work were housewives, of moderate socio-economic status.

In our study, there was a highly statistically significant correlation between the presence of TTS and FIQ scores of FM patients $(p<0.001)$. Moreover, comparison between FM patients with and without TTS as regards their level of impairment, presented by the FIQ score, revealed highly statistically significant difference, with a higher mean value in FM patients with TTS $(p<0.001)$.

The correlation between the presence of TTS and the FIQ score of FM patients and the presence of higher FIQ score in patients with CTS emphasizes the fact that TTS is an essential cause of impairment in FM patients, whether it is or is not a part of the disease.
Besides, we agree with $\mathrm{Yo}$ and $\mathrm{Na}(4)$, in that the presence of TTS may be masked by the symptoms of FM. This was established by history and clinical examination of our patients, as 17 patients were suffering from paresthesia and electrophysiological evidence of TTS was present only in 8 patients. So, patients with FM should be assessed by appropriate electrophysiological tests. Appropriate and effective therapeutic interventions for TTS should be applied to improve quality of life of patients with FM.

From the previous discussion we conclude that TTS is frequent among patients with FM than the normal population. Electrophysiological examination should be performed for every patient. Correlations between the severity of TTS and the FIQ scores should be performed to clarify the main causes of impairment in patients with FM. So, management of TTS in these patients should be tailored to minimalize impairments.

\section{References}

1. Firestein GS, Gabriel SE, McInnes IB, O'Dell JR. Kelley and Firestein's textbook of rheumatology. 2017.

2. Ofluoglu D, Berker N, Guven Z, Canbulat N, Yilmaz IT, et al. (2005) Quality of life in patients with fibromyalgia syndrome and rheumatoid arthritis. Clinical rheumatology 24: 490-492.

3. Wolfe F1, Ross K, Anderson J, Russell IJ, Hebert L (1995) The prevalence and characteristics of fibromyalgia in the general population. Arthritis Rheum 38: 19-28. [Crossref]

4. Jo YS, Na SJ (2017) Tarsal tunnel syndrome in patients with fibromyalgia. Journal of the Neurological Sciences 381: 469.

5. Antoniadis G, Scheglmann K (2008) Posterior tarsal tunnel syndrome: diagnosis and treatment. Dtsch Arztebl Int 105: 776-781. [Crossref]

6. Martin S, Chandran A, Zografos L, Zlateva G (2009) Evaluation of the impact of fibromyalgia on patients' sleep and the content validity of two sleep scales. Health Qual Life Outcomes 7: 64. [Crossref]

7. Wolfe F (2010) New American College of Rheumatology criteria for fibromyalgia: a twenty-year journey. Arthritis Care Res (Hoboken) 62: 583-584. [Crossref]

8. Schwieterman B, Haas D, Columber K, Knupp D, Cook C (2013) Diagnostic accuracy of physical examination tests of the ankle/foot complex: a systematic review. Int $J$ Sports Phys Ther 8: 416-426. 
9. Verbraecken J, Van de Heyning P, De Backer W, Van Gaal L (2006) Body surface area in normal-weight, overweight, and obese adults. A comparison study. Metabolism 55: 515-524. [Crossref]

10. Feng Y, Schlösser FJ, Sumpio BE (2009) The Semmes Weinstein monofilament examination as a screening tool for diabetic peripheral neuropathy. Journal of Vascular Surgery 50: 675-682.e1.

11. El-Naby MA, Hefny MA, Fahim AE, Awadalla MA (2013) Validation of an adapted arabic version of fibromyalgia syndrome impact questionnaire. Rheumatology international 33: 2561-2567.

12. Oh SJ, Sarala PK, Kuba T, Elmore RS (1979) Tarsal tunnel syndrome: electrophysiological study. Ann Neurol 5: 327-330.

13. Buschbacher RM (1999) Tibial nerve conduction to the flexor digiti minimi brevis. $\mathrm{Am}$ J Phys Med Rehabil 78: S21-25. [Crossref]

14. Fu R, DeLisa JA, Kraft GH (1980) Motor nerve latencies through the tarsal tunnel in normal adult subjects: standard determinations corrected for temperature and distance. Archives of physical medicine and rehabilitation 61: 243-248.
15. Weiss J, Weiss LD, Silver JK (2016) Easy EMG: a guide to performing nerve conduction studies and electromyography.

16. Van den Bergh PY, Hadden RD, Bouche P, Cornblath DR, Hahn A, et al. (2010) European Federation of Neurological Societies/Peripheral Nerve Society guideline on management of chronic inflammatory demyelinating polyradiculoneuropathy: report of a joint task force of the European Federation of Neurological Societies and the Peripheral Nerve Society - first revision. European journal of neurology 17: 356-363.

17. Uncini A, Kuwabara S (2012) Electrodiagnostic criteria for Guillain-Barre syndrome: a critical revision and the need for an update. Clin Neurophysiol 123: 1487-1495.

18. Jones J, Rutledge DN, Jones KD, Matallana L, Rooks DS (2008) Self-assessed physical function levels of women with fibromyalgia: a national survey. Womens Health Issues 18: 406-412.

19. Shookster L, Falke GI, Ducic I, Maloney CT Jr, Dellon AL (2004) Fibromyalgia and Tinel's sign in the foot. J Am Podiatr Med Assoc 94: 400-403. [Crossref]

Copyright: $\odot 2018$ Hefny MA. This is an open-access article distributed under the terms of the Creative Commons Attribution License, which permits unrestricted use, distribution, and reproduction in any medium, provided the original author and source are credited. 